\title{
Complex B-splines and Hurwitz zeta functions
}

\author{
B. Forster, R. Garunkštis, P. Massopust and J. Steuding
}

\begin{abstract}
We characterize nonempty open subsets of the complex plane where the sum $\zeta(s, \alpha)+$ $e^{ \pm i \pi s} \zeta(s, 1-\alpha)$ of Hurwitz zeta functions has no zeros in $s$ for all $0 \leqslant \alpha \leqslant 1$. This problem is motivated by the construction of fundamental cardinal splines of complex order $s$.
\end{abstract}

\section{Introduction}

Cardinal B-splines of complex order or, for short, complex B-splines, are a natural extension of the classical Curry-Schoenberg (polynomial) B-splines where the integral order $n$ is replaced by a complex number $s$. More precisely, complex B-splines $B_{s}: \mathbb{R} \rightarrow \mathbb{C}$ are defined in the Fourier domain by

$$
\mathcal{F}\left(B_{s}\right)(\omega):=\int_{\mathbb{R}} B_{s}(t) e^{-i \omega t} d t:=\left(\frac{1-e^{-i \omega}}{i \omega}\right)^{s},
$$

for $\operatorname{Re} s>1$. Setting $\Omega: \mathbb{R} \rightarrow \mathbb{C}, \omega \mapsto\left(1-e^{-i \omega}\right) / i \omega$, one notices that graph $\Omega \cap\{y \in \mathbb{R}$ : $y<0\}=\emptyset$, implying that complex B-splines reside on the main branch of the complex logarithm and are thus well-defined.

Complex B-splines were first investigated in [3], where several important properties of these splines were shown. For instance, in the time domain $B_{s}$ is a piecewise polynomial of complex degree $s-1$

$$
B_{s}(x)=\frac{1}{\Gamma(z)} \sum_{k \geqslant 0}(-1)^{k}\left(\begin{array}{l}
s \\
k
\end{array}\right)(x-k)_{+}^{s-1} \quad \text { for all } x \in \mathbb{R}
$$

with fast decay at infinity:

$$
B_{s}(x) \in \mathcal{O}\left(x^{-m}\right) \quad \text { for } m<\operatorname{Re} s+1,|x| \rightarrow \infty .
$$

Moreover, complex B-splines are elements of $L^{1}(\mathbb{R}) \cap L^{2}(\mathbb{R})$ satisfying recursion formulas similar to those of the classical polynomial B-splines, are scaling functions, and generate multiresolution analyses and wavelets. But in general, they do not have compact support. Furthermore, complex B-splines relate in a natural fashion to difference and differential operators of fractional order. For further properties and relationships to Dirichlet averages and fractional derivatives and integrals we refer the interested reader to $[\mathbf{4}, \mathbf{5}]$.

\section{The interpolation problem for splines of complex order}

In order to solve the cardinal spline interpolation problem using the classical Curry-Schoenberg splines $[\mathbf{1}, \mathbf{6}]$, one constructs a fundamental cardinal spline function that is a linear bi-infinite combination of polynomial B-splines $B_{n}$ of fixed order $n \in \mathbb{N}$ which interpolates the data set $\left\{\delta_{m, 0}: m \in \mathbb{Z}\right\}$. More precisely, one solves the bi-infinite system

$$
\sum_{k \in \mathbb{Z}} c_{k}^{(n)} B_{n}\left(\frac{n}{2}+m-k\right)=\delta_{m, 0}, \quad m \in \mathbb{Z},
$$

Received 30 July 2012.

2010 Mathematics Subject Classification 11M35, 65D07 (primary), 11M26, 65D05 (secondary). 
for the sequence $\left\{c_{k}^{(n)}: k \in \mathbb{Z}\right\}$. The left-hand side of (2) defines the fundamental cardinal spline $L_{n}: \mathbb{R} \rightarrow \mathbb{R}$ of order $n \in \mathbb{N}$. A formula for $L_{n}$ is given in terms of its Fourier transforms by

$$
\widehat{L}_{n}(\omega)=\frac{\left(\widehat{B}_{n}(\bullet+n / 2)\right)(\omega)}{\sum_{k \in \mathbb{Z}}\left(\widehat{B}_{n}(\bullet+n / 2)\right)(\omega+2 \pi k)} .
$$

Using the Euler-Frobenius polynomials associated with the B-splines $B_{n}$, one can show that the denominator in (3) does not vanish on the unit circle $|z|=1$, where $z=e^{-i \omega}$. For details, see $[\mathbf{1}, \mathbf{6}]$.

One of the goals in the theory of splines of complex order is to construct a fundamental cardinal spline $L_{s}: \mathbb{R} \rightarrow \mathbb{C}$ of complex order $s=\sigma+i t$ of the form

$$
L_{s}:=\sum_{k \in \mathbb{Z}} c_{k}^{(s)} B_{s}(\bullet-k),
$$

satisfying the interpolation problem

$$
L_{s}(m)=\delta_{m, 0}, \quad m \in \mathbb{Z},
$$

for an appropriate bi-infinite sequence $\left\{c_{k}^{(s)}: k \in \mathbb{Z}\right\}$ and for appropriate $s$ belonging to some nonempty open subset of $\mathbb{C}$.

Remark 1. Note that, unlike in the case of the Curry-Schoenberg B-splines [6], which are even functions, we consider the nonsymmetric, that is, shifted versions $B_{s}$. The reason is that the function $\Omega$ defined above would cross the negative real axis if $\Omega$ were equal to sinc $(\omega / 2)$ resulting in the loss of well-definedness of complex B-splines. We will see that our case excludes interpolation of B-splines with integer order $s=2 n+1, n \in \mathbb{N}_{0}$. This case, however, is covered by the Schoenberg's symmetric B-splines: there are interpolating B-splines of order $2 n+1$.

Taking the Fourier transform of (4) and (5), and eliminating the expression containing the unknowns $\left\{c_{k}^{(s)}: k \in \mathbb{Z}\right\}$, a formula for $\widehat{L}_{s}$ similar to (3) is, at first, formally obtained:

$$
\widehat{L}_{s}(\omega)=\frac{\widehat{B}_{s}(\omega)}{\sum_{k \in \mathbb{Z}} \widehat{B}_{s}(\omega+2 \pi k)} .
$$

Inserting (1) into the above expression for $\widehat{L}_{s}$ and simplifying with the convention $\arg t=-\pi$ for $t<0$ yields

$$
\widehat{L}_{s}(\omega)=\frac{1 / \omega^{s}}{\sum_{k \in \mathbb{Z}} 1 /(\omega+2 \pi k)^{s}}, \quad \operatorname{Re} s>1 .
$$

(The choice $\arg t=\pi$ would yield the right-hand side formula with $-\omega$ instead of $\omega$.) Since the denominator of (6) is $2 \pi$-periodic, we may assume without loss of generality that $\omega \in[0,2 \pi]$, and since $\widehat{L}_{s}(0)=1$ and $\widehat{L}_{s}(2 \pi)=0$, it suffices to consider $0<\omega<2 \pi$. Setting $\alpha:=\omega /(2 \pi)$, the sum in the above denominator can be rewritten in the form

$$
\begin{aligned}
\sum_{k \in \mathbb{Z}} \frac{1}{(k+\alpha)^{s}} & =\sum_{k=0}^{\infty} \frac{1}{(k+\alpha)^{s}}+\sum_{k=0}^{\infty} \frac{1}{(\alpha-1-k)^{s}} \\
& =\sum_{k=0}^{\infty} \frac{1}{(k+\alpha)^{s}}+e^{-i \pi s} \sum_{k=0}^{\infty} \frac{1}{(k+1-\alpha)^{s}} \\
& =\zeta(s, \alpha)+e^{-i \pi s} \zeta(s, 1-\alpha),
\end{aligned}
$$

where we take the principal value of the multi-valued function $e^{-i \pi(\bullet)}$ and where $\zeta(s, \alpha)$ denotes the Hurwitz zeta function to the parameter $0<\alpha<1$.

The aim of this article is to characterize nonempty open subsets of the complex plane for which (7) has no zeros for all $\alpha \in$ ] 0,1 [. For these parameters $s$ the interpolation property (5) 
is satisfied. To this end, in the next section, we first investigate the case of fundamental Bsplines $L_{\sigma}$ of real order $\sigma \in \mathbb{R}$. In $\S 4$ we find trajectories of nonreal zeros. Then in the following two sections we derive rectangular and crescent-shaped regions where (7) is zero free.

\section{Special case: fundamental B-splines of real order}

In this section, it is our goal to construct a fundamental cardinal spline $L_{\sigma}: \mathbb{R} \rightarrow \mathbb{C}$ of real order $\sigma \in \mathbb{R}_{+}$. To show that in this special case the denominator in (6) does not vanish, that is, that $L_{\sigma}$ is well-defined, it suffices to find conditions on $\sigma$ so that the function (7)

$$
\zeta(\sigma, \alpha)+e^{-i \pi \sigma} \zeta(\sigma, 1-\alpha)
$$

has no zeros for all $\alpha \in(0,1)$. To this end, we use the following theorem by Spira [7].

Theorem 3.1. If $\operatorname{Re} s \geqslant 1+\alpha$, then $\zeta(s, \alpha) \neq 0$.

Choosing a real $\sigma \geqslant 2$ guarantees that for $0<\alpha<1$ neither $\zeta(\sigma, \alpha)$ nor $\zeta(\sigma, 1-\alpha)$ has zeros in $[2, \infty) \subset \mathbb{R}$. Firstly, assume that $0<\alpha<\frac{1}{2}$. Then

$$
\begin{aligned}
\left|e^{-i \pi \sigma} \zeta(\sigma, 1-\alpha)\right| & \leqslant \sum_{k=0}^{\infty} \frac{1}{(k+1-\alpha)^{\sigma}} \\
& =\frac{1}{(1-\alpha)^{\sigma}}+\sum_{k=1}^{\infty} \frac{1}{(k+1-\alpha)^{\sigma}} \\
& \leqslant \frac{1}{(1-\alpha)^{\sigma}}+\sum_{k=1}^{\infty} \frac{1}{(k+\alpha)^{\sigma}} \\
& <\frac{1}{\alpha^{\sigma}}+\sum_{k=1}^{\infty} \frac{1}{(k+\alpha)^{\sigma}}=|\zeta(\sigma, \alpha)| .
\end{aligned}
$$

For $\frac{1}{2}<\alpha<1$, we obtain in a similar fashion that $|\zeta(\sigma, \alpha)|<\left|e^{-i \pi \sigma} \zeta(\sigma, 1-\alpha)\right|$. Hence,

$$
\left|\zeta(\sigma, \alpha)+e^{-i \pi \sigma} \zeta(\sigma, 1-\alpha)\right| \geqslant|| \zeta(\sigma, \alpha)|-| e^{-i \pi \sigma} \zeta(\sigma, 1-\alpha) \|>0,
$$

for all $\alpha \in(0,1) \backslash\left\{\frac{1}{2}\right\}$.

Secondly, assume that $\alpha=\frac{1}{2}$ and that $\sigma \geqslant 2$. Then the second factor in $\left(1+e^{-i \pi \sigma}\right) \zeta\left(\sigma, \frac{1}{2}\right)$ is nonzero by Theorem 3.1. To guarantee that the first factor is also nonzero, $\sigma$ must not belong to $2 \mathbb{N}+1$. Thus, for all $0<\alpha<1$ and for all $\sigma \in \mathbb{R}$ with $\sigma \geqslant 2$ and $\sigma \notin 2 \mathbb{N}+1$, $\zeta(\sigma, \alpha)+e^{-i \pi \sigma} \zeta(\sigma, 1-\alpha) \neq 0$.

REMARK 2. Note that the conditions on $\sigma$ are independent of the branch chosen for the multi-valued function $e^{-i \pi(\bullet)}$.

Let $\mathfrak{R}:=\{x \in \mathbb{R}: x \geqslant 2 \wedge x \notin 2 \mathbb{N}+1\}$. Then, since $B_{\sigma} \in L^{1}(\mathbb{R}) \cap L^{2}(\mathbb{R})$, the fundamental cardinal spline $L_{\sigma}$ of real order $\sigma$ with $\sigma \in \mathfrak{R}$ is also an element of $L^{1}(\mathbb{R}) \cap L^{2}(\mathbb{R})$ and, in particular, continuous on $\mathbb{R}$. Figure 1 shows some of the splines $L_{\sigma}$ in the frequency domain and Figure 2 in the time domain.

\section{Nonreal zeros}

To find nonempty open subsets of the complex plane for which (7) has no zeros, we consider a more general sum of Hurwitz zeta functions. For $s=\sigma+i t$ and a real parameter $\alpha \in(0,1)$ define

$$
f_{ \pm}(s, \alpha):=\zeta(s, \alpha)+e^{ \pm i \pi s} \zeta(s, 1-\alpha)
$$


(a)

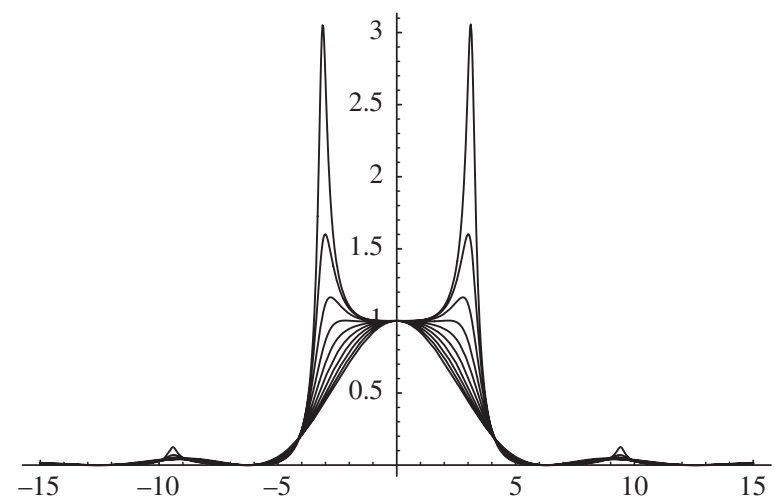

(b)

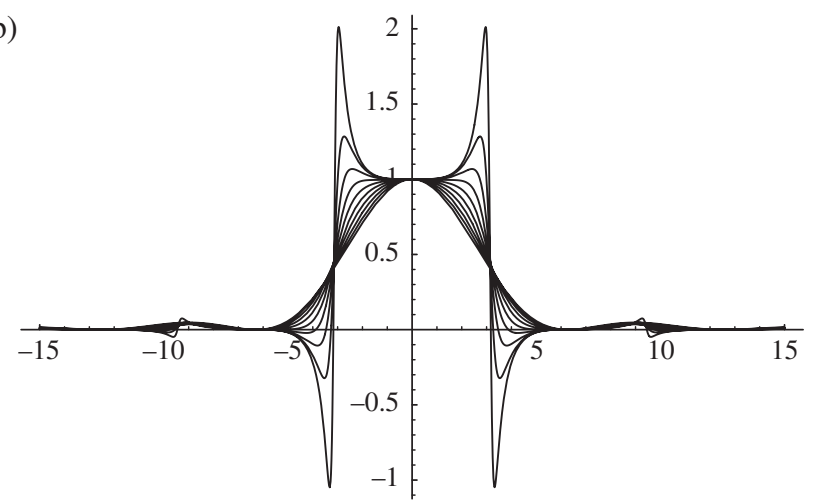

(c)

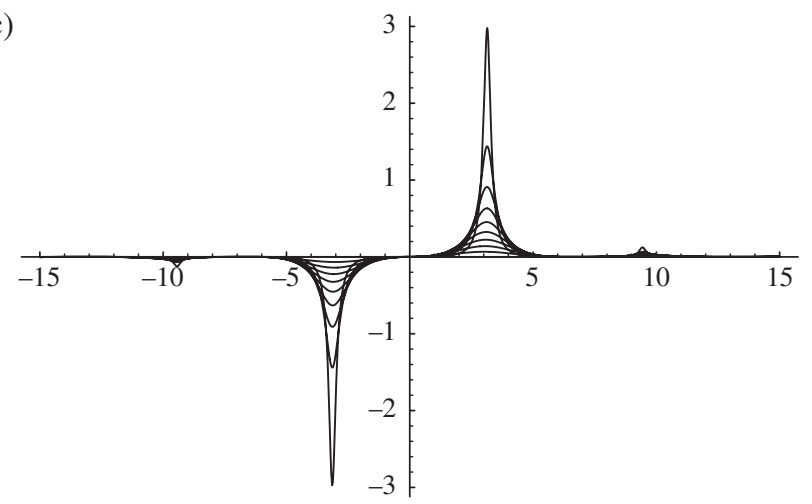

FiguRE 1. The interpolating spline $\widehat{L}_{\sigma}$ in the frequency domain for the parameters $\sigma=2.0,2.1, \ldots, 2.9$. (a) Absolute value. (b) Real part. (c) Imaginary part. For $\sigma=2.9$, a value close to the excluded exponent $\sigma=3$, large values in a neighborhood of the singularities at $\pm \pi(2 \mathbb{Z}+1)$ appear.

For $\sigma>1$, the Hurwitz zeta function to the parameter $\alpha \in(0,1]$ can be extended by analytic continuation to all of $\mathbb{C}$ except for a simple pole at $s=1$. (See, for example, [2].) Obviously, $f_{ \pm}$ vanishes at $s=2 n+1$ with $n \in \mathbb{N}$ for $\alpha=\frac{1}{2}$. Further zeros can be found along the trajectories as $\alpha$ varies. This is illustrated in Figure 3 .

The function $f_{ \pm}$is analytic in $s$ and $\alpha$ for $\sigma>1$ and $0<\alpha<1$. The computation of these zero trajectories is based on the numerical solutions of the differential equation

$$
\frac{\partial z(\alpha)}{\partial \alpha}=-\frac{\partial f_{ \pm}(z, \alpha) / \partial \alpha}{\partial f_{ \pm}(z, \alpha) / \partial z}
$$


(a)

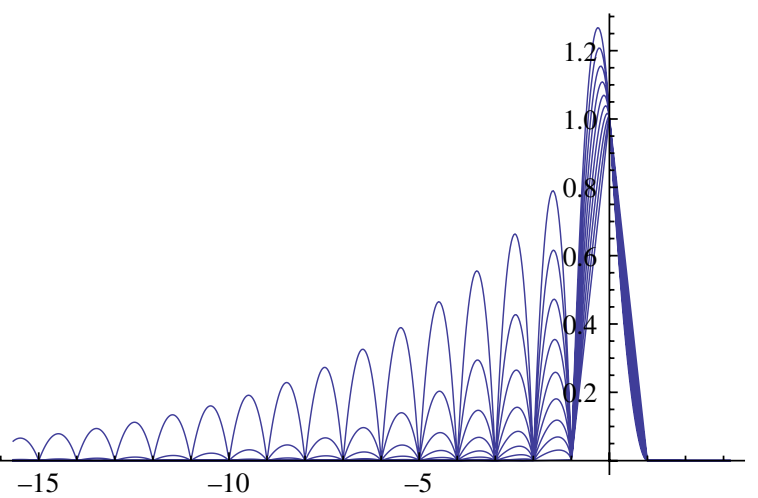

(b)

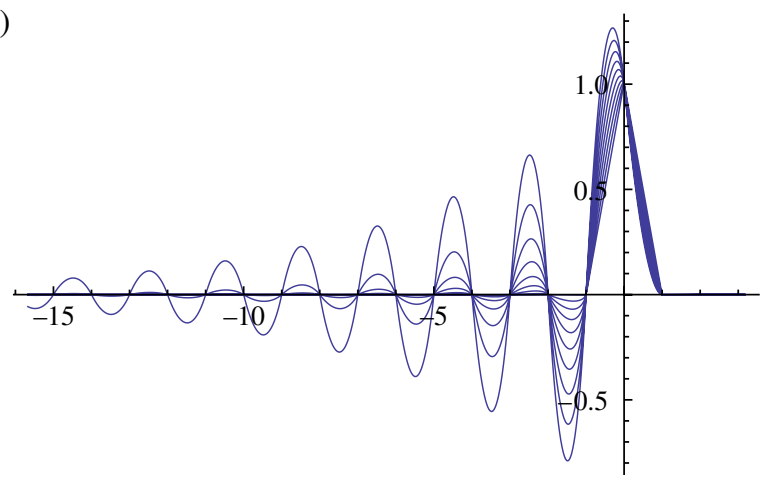

Figure 2. The interpolating spline $L_{\sigma}$ in the time domain for the parameters $\sigma=2.0,2.1, \ldots, 2.9$. (a) Absolute value. (b) Real part. The imaginary part vanishes for all these parameters $\sigma$. For $\sigma=2$ we have the classical case of linear interpolation.

where $z=z(\alpha), f_{ \pm}(z(\alpha), \alpha)=0$, and the initial conditions are provided by the zeros $s=2 n+1$, $n \in \mathbb{N}$, and $\alpha=\frac{1}{2}$. Since the accuracy of these computations was not controlled, they cannot be regarded as a proof for the existence of nonreal zeros.

However, we can prove the existence of zeros off the real axis. To this end, note that the function $f_{ \pm}(s, \alpha)$ depends continuously on $\alpha$ for values of $\alpha$ near $\frac{1}{2}$. In fact, for all $\varepsilon>0$, there exists a $\delta>0$ such that for all $\alpha$ with $\left|\alpha-\frac{1}{2}\right|<\delta$

$$
\left|f_{ \pm}(s, \alpha)-f_{ \pm}\left(s, \frac{1}{2}\right)\right|<\varepsilon .
$$

Let $s$ be on a circle $|s-2 n-1|=r$ for $n \in \mathbb{N}$ and some $0<r<2$. Then, according to Spira's Theorem 3.1, the function $f_{ \pm}\left(s, \frac{1}{2}\right)$ does not vanish. In particular, $\left|f_{ \pm}\left(s, \frac{1}{2}\right)\right|>0$. We now set $\varepsilon:=\left|f_{ \pm}\left(s, \frac{1}{2}\right)\right|>0$, which for $\left|\alpha-\frac{1}{2}\right|<\delta$, implies the inequality

$$
\left|f_{ \pm}(s, \alpha)-f_{ \pm}\left(s, \frac{1}{2}\right)\right|<\left|f_{ \pm}\left(s, \frac{1}{2}\right)\right|,
$$

for all $s$ on the circle $|s-2 n-1|=r$, for $n \in \mathbb{N}$ and some $0<r<2$.

It follows from Rouché's theorem that for all $\left|\alpha-\frac{1}{2}\right|<\delta$ the functions $f_{ \pm}(s, \alpha)$ and $f_{ \pm}\left(s, \frac{1}{2}\right)$ have the same number of zeros inside the disk of radius $r$ centered at $s=2 n+1$. As $f_{ \pm}\left(2 n+1, \frac{1}{2}\right)=0$, the function $f_{ \pm}(s, \alpha)$ has at least one zero in this disk. However, such a zero cannot be real since for real $s>1$

$$
\frac{\zeta(s, 1-\alpha)}{\zeta(s, \alpha)}=-\exp ( \pm i \pi s)
$$

equals one if and only if $s=2 n+1$ for some $n \in \mathbb{N}$. Thus we have proved the following theorem. 


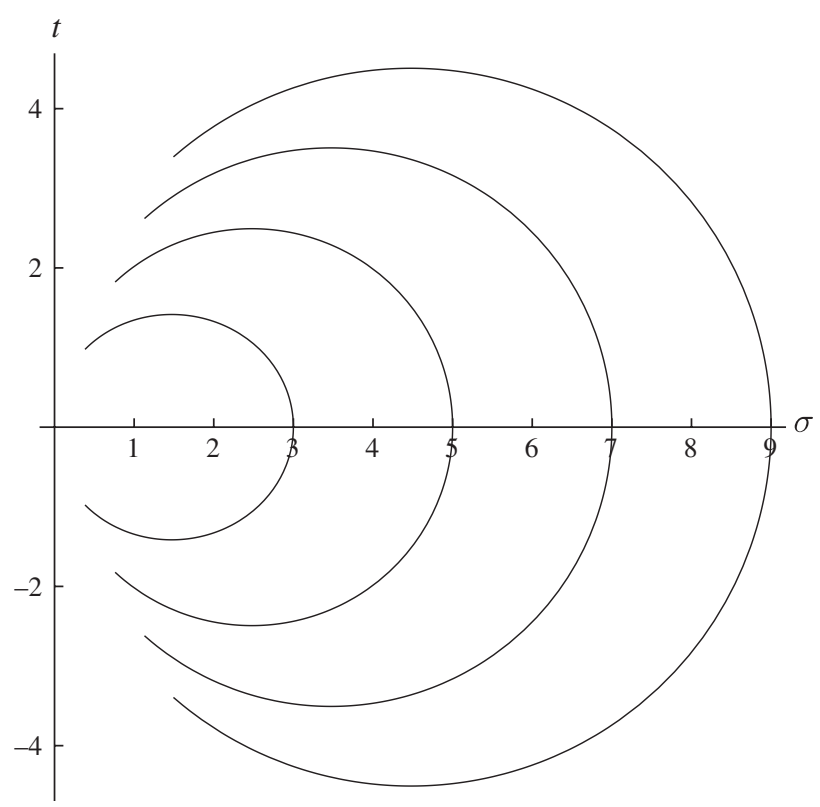

Figure 3. Zero trajectories $z_{j}(\alpha)$ in the $\sigma+i$-plane according to equation (9) for $j=1,2,3$, 4, defined by $f_{+}\left(z_{j}(\alpha), \alpha\right)=0$, for $0.001<\alpha<0.999$. As starting points, we chose the zeros on the real axis and $z_{1}(1 / 2)=3, \ldots, z_{4}(1 / 2)=9$.

Theorem 4.1. For any $n \in \mathbb{N}$, there exist values of $\alpha$, not equal to $\frac{1}{2}$, so that the function $f_{ \pm}(s, \alpha)$ has a nonreal zero $s_{0}$ in a neighborhood of $s=2 n+1$.

In the following section we establish the existence of nonempty open sets on which $f_{ \pm}(s, \alpha)$ does not vanish as a function of $s$ for all values of $\alpha \in(0,1)$. Actually, we obtain such a nonvanishing of $f_{ \pm}(2 n+z, \alpha)$ for all $\alpha \in(0,1)$ and sufficiently large $n$ with $z$ from a certain small rectangle to be specified below. Comparing with Figure 3, these rectangles are nested between the zero trajectories.

\section{Zero-free rectangles}

We now identify rectangles in the complex $s$-plane, in which $f_{ \pm}(s, \alpha)$ does not vanish for all $0<\alpha<1$.

First, we observe that a functional equation holds for $f_{ \pm}$with respect to the parameter $0<\alpha<1$ :

$$
f_{ \pm}(s, 1-\alpha)=e^{ \pm i \pi s} f_{\mp}(s, \alpha) .
$$

Hence, we have $f_{ \pm}(s, \alpha) \neq 0$ for $0<\alpha<1$ if and only if $f_{ \pm}(s, \alpha) \neq 0$ for $0<\alpha \leqslant \frac{1}{2}$. Therefore, in the following we may assume that $0<\alpha<\frac{1}{2}$ and $\sigma>1$.

For $\sigma>1$, we have that

$$
\zeta(\sigma)-1=\sum_{k=2}^{\infty} k^{-\sigma}<\int_{1}^{\infty} u^{-\sigma} d u=\frac{1}{\sigma-1}<\sum_{k=1}^{\infty} k^{-\sigma}=\zeta(\sigma),
$$

where $\zeta(\sigma)$ denotes the Riemann zeta function. Therefore,

$$
\frac{1}{\sigma-1}<\zeta(\sigma)<1+\frac{1}{\sigma-1}
$$


for the same range of $\sigma$. It is not difficult to deduce from the latter inequalities that

$$
\sum_{k=2}^{\infty} \frac{1}{(k+\alpha)^{\sigma}}<\frac{1}{\sigma-1} \text { and } \sum_{k=2}^{\infty} \frac{1}{(k+1-\alpha)^{\sigma}}<\frac{1}{\sigma-1} .
$$

We define

$$
g_{ \pm}(s, \alpha):=\alpha^{s} f_{ \pm}(s, \alpha),
$$

which has the same zero as $f_{ \pm}(s, \alpha)$ for $s \in \mathbb{C}$ and $0<\alpha<1$. We approximate $g_{ \pm}(s, \alpha)$ by $1+A(s, \alpha)$, where

$$
A(s, \alpha):=e^{ \pm i \pi s}\left(\frac{\alpha}{1-\alpha}\right)^{s} .
$$

It follows from the above estimates that

$$
\begin{aligned}
\left|g_{ \pm}(s, \alpha)-(1+A(s, \alpha))\right|= & \left|\alpha^{s}\left(\zeta(s, \alpha)+e^{ \pm i \pi s} \zeta(s, 1-\alpha)\right)-\left(1+e^{ \pm i \pi s}\left(\frac{\alpha}{1-\alpha}\right)^{s}\right)\right| \\
= & \mid \alpha^{s}\left(\frac{1}{\alpha^{s}}+\frac{1}{(1+\alpha)^{s}}+\sum_{k=2}^{\infty} \frac{1}{(k+\alpha)^{s}}\right. \\
& \left.+e^{ \pm i \pi s}\left(\frac{1}{(1-\alpha)^{s}}+\frac{1}{(2-\alpha)^{s}}+\sum_{k=2}^{\infty} \frac{1}{(k+1-\alpha)^{s}}\right)\right) \\
& -1-e^{ \pm i \pi s}\left(\frac{\alpha}{1-\alpha}\right)^{s} \mid \\
\leqslant & \left(\frac{\alpha}{1+\alpha}\right)^{\sigma}+e^{\mp \pi t}\left(\frac{\alpha}{2-\alpha}\right)^{\sigma}+\frac{1+e^{\mp \pi t}}{\sigma-1} \alpha^{\sigma} .
\end{aligned}
$$

Now, let $s=2 n+2+z$, where $z=x+i y$ with $y \neq 0, n \in \mathbb{N}$. Note that, for $0<\alpha \leqslant \frac{1}{2}$,

$$
\frac{\alpha}{1+\alpha} \leqslant \frac{1}{3} \text { and } \quad \frac{\alpha}{1+1-\alpha} \leqslant \frac{1}{3} .
$$

Hence, inequality (13) can be further estimated by

$$
\left|g_{ \pm}(2 n+2+z, \alpha)-(1+A)\right| \leqslant B
$$

where

$$
A:=A(2 n+2+z, \alpha)=e^{ \pm i \pi z}\left(\frac{\alpha}{1-\alpha}\right)^{2 n+2+z}
$$

and

$$
B:=B(2 n+2+z):=\left(1+e^{\mp \pi y}\right)\left(3^{-2 n-2-x}+\frac{2^{-2 n-2-x}}{2 n+1+x}\right) .
$$

For later use we also compute

$$
\operatorname{Re} A=\left(\frac{\alpha}{1-\alpha}\right)^{2 n+2+x} e^{\mp \pi y} \cos \left( \pm \pi x+y \ln \frac{\alpha}{1-\alpha}\right) .
$$

Now we aim at describing a set $S \subset \mathbb{C}$ depending on $n \in \mathbb{N}$, such that

$$
g_{ \pm}:=g_{ \pm}(2 n+2+z, \alpha) \neq 0, \quad \forall z \in S \quad \text { and } \quad \forall 0<\alpha<\frac{1}{2} .
$$

However, in considering (16) for $\alpha$ close to $\frac{1}{2}$ this would result in restricting $z$ to a small neighborhood of the real axis. Therefore, we split the procedure into two steps.

Step 1. We find an interval $I$ for $\alpha$ close to $\frac{1}{2}$ such that $\operatorname{Re} A>0$. Then we choose a rectangle $S$, to be determined later in Step 2, so that $B<1$. 
Step 2. For the remaining values of $\alpha \notin I$, we show that $|A|+B<1$ is satisfied for the rectangle $S$. By (14) and the weaker formulation

$$
\left|g_{ \pm}-1\right| \leqslant|A|+B<1
$$

this implies that $g_{ \pm}$is close to 1 and therefore $g_{ \pm} \neq 0$.

To this end, let $0<X<\frac{1}{2}$ and $0<Y$. Denote by

$$
S:=S(n, X, Y):=\{s \in \mathbb{C}: s=2 n+2+z,|\operatorname{Re} z| \leqslant X,|\operatorname{Im} z| \leqslant Y\}
$$

the rectangle in the complex $s$-plane of width $X$ and height $Y$ and with center $2 n+2, n \in \mathbb{N}$.

We start with step 1 . In fact, from equation (16) we see that $\operatorname{Re} A \geqslant 0$ if the cosine term is positive. This is the case for

$$
-\frac{\pi}{2} \leqslant \pm \pi x+y \ln \frac{\alpha}{1-\alpha} \leqslant \frac{\pi}{2}
$$

which is equivalent to

$$
\left| \pm \pi x+y \ln \frac{\alpha}{1-\alpha}\right| \leqslant \frac{\pi}{2}
$$

Due to the inverse triangle inequality, this estimate is implied by

$$
| \pm \pi x|+\left|y \ln \frac{\alpha}{1-\alpha}\right| \leqslant \frac{\pi}{2},
$$

which, on the other hand, is implied by $s \in S$ and

$$
\pi X+Y\left|\ln \frac{\alpha}{1-\alpha}\right| \leqslant \frac{\pi}{2} .
$$

This is equivalent to $s \in S$ and

$$
\frac{\pi X-\pi / 2}{Y} \leqslant \ln \frac{\alpha}{1-\alpha} \leqslant \frac{\pi / 2-\pi X}{Y} .
$$

The left-hand inequality is satisfied if $s \in S$ and

$$
C(X, Y):=\frac{\exp ((\pi X-\pi / 2) / Y)}{1+\exp ((\pi X-\pi / 2) / Y)}<\alpha \leqslant \frac{1}{2},
$$

since the right-hand inequality holds trivially for $X<\frac{1}{2}$. In view of this and expression (14), it follows that $g_{ \pm}(s, \alpha) \neq 0$ provided $X$ and $Y$ are chosen so that

$$
B<1 \text { for all } s \in S \text { and } C(X, Y)<\alpha \leqslant \frac{1}{2} \text {. }
$$

Step 2 is now easy. For $g_{ \pm} \neq 0$ we need to show that

$$
|A|+B<1 \text { for } 0<\alpha \leqslant C(X, Y) \text { and for all } s \in S .
$$

Note that the term $B$ does not depend on $\alpha$ and that the function $\alpha \mapsto \alpha /(1-\alpha)$ is increasing for $0<\alpha \leqslant \frac{1}{2}$. If

$$
J(n, X, Y):=\left(\frac{C(X, Y)}{1-C(X, Y)}\right)^{2 n+2-X} e^{\pi Y}+\left(1+e^{\pi Y}\right)\left(3^{-2 n-2+X}+\frac{2^{-2 n-2+X}}{2 n+1-X}\right)<1,
$$

then (19) holds. Employing the above expression for $C(X, Y), J(n, X, Y)$ simplifies to

$$
J(n, X, Y)=e^{\pi\left(X-\frac{1}{2}\right)(2 n+2-X) Y^{-1}} e^{\pi Y}+\left(1+e^{\pi Y}\right)\left(3^{-2 n-2+X}+\frac{2^{-2 n-2+X}}{2 n+1-X}\right) .
$$

Note that the first exponential term is less than one only if $X<\frac{1}{2}$. Thus, both inequalities (18) and (19) are satisfied if $s \in S$ such that $J(n, X, Y)<1$. 
We now show that for given $n \in \mathbb{N}$ and $0<X<\frac{1}{2}$, there always exists a $Y$ so that $J(n, X, Y)<1$. For this purpose, observe that

$$
3^{-2 n-2+X}+\frac{2^{-2 n-2+X}}{2 n+1-X}<\left(\frac{1}{2}\right)^{2 n+2-1 / 2}+\left(\frac{1}{2}\right)^{2 n+2-1 / 2}=\left(\frac{1}{2}\right)^{2 n+1 / 2} .
$$

Hence, $J(n, X, Y)<1$ if, for instance,

$$
e^{\pi\left(X-\frac{1}{2}\right)(2 n+2-X) Y^{-1}} e^{\pi Y}<\frac{1}{2} \quad \text { and } \quad\left(1+e^{\pi Y}\right)\left(\frac{1}{2}\right)^{2 n+1 / 2}<\frac{1}{2} .
$$

The latter inequality holds if

$$
Y<\frac{1}{\pi} \ln \left(2^{2 n-1 / 2}-1\right)=: Y_{1}
$$

and the former if

$$
\pi\left[\frac{\left(X-\frac{1}{2}\right)(2 n+2-X)}{Y}+Y\right]<\ln \frac{1}{2} .
$$

Solving this quadratic inequality for $Y$ yields

$$
Y<\frac{\ln \frac{1}{2}}{2 \pi}+\sqrt{\left(\frac{\ln \frac{1}{2}}{2 \pi}\right)^{2}+\left(X-\frac{1}{2}\right)(2 n+2-X)}=: Y_{2}
$$

Therefore, choosing

$$
Y<\min \left\{Y_{1}, Y_{2}\right\}
$$

implies $J(n, X, Y)<1$. Note that in this case, the sequence $\{J(m, X, Y): m \geqslant n\}$ is decreasing.

The above arguments now yield the following result.

Theorem 5.1. Let $0<X<\frac{1}{2}$ and $Y>0$. Assume that $s \in S(n, X, Y), n \in \mathbb{N}$, and $0<\alpha<1$. Then $f_{ \pm}(s, \alpha) \neq 0$ provided $J(n, X, Y)<1$. Moreover, $J(m, X, Y)<J(n, X, Y)$ if $m>n$, $m \in \mathbb{N}$.

For an illustration of Theorem 5.1, see Figure 4.

\section{Crescent-shaped zero-free regions}

Again, in view of (10), we consider only $0<\alpha \leqslant \frac{1}{2}$. Recall that for $s=\sigma+i t$, we have by (12), (13), and (15)

$$
\begin{aligned}
\left|\alpha^{s} f_{ \pm}(s, \alpha)-\left(1+e^{ \pm i \pi s}\left(\frac{\alpha}{1-\alpha}\right)^{s}\right)\right| & \leqslant\left(\frac{\alpha}{1+\alpha}\right)^{\sigma}+e^{\mp \pi t}\left(\frac{\alpha}{2-\alpha}\right)^{\sigma}+\frac{1+e^{\mp \pi t}}{\sigma-1} \alpha^{\sigma} \\
& \leqslant\left(1+e^{\mp \pi t}\right)\left(3^{-\sigma}+\frac{2^{-\sigma}}{\sigma-1}\right) .
\end{aligned}
$$

For a better understanding of the zero trajectories of $f_{ \pm}(s, \alpha)$ described in Figure 3, we consider the zero trajectories of the approximation term in expression (20). These trajectories are circles in the $s$-plane; namely, we have

$$
1+e^{ \pm i \pi s}\left(\frac{\alpha}{1-\alpha}\right)^{s}=0 \quad \text { for } 0<\alpha<1
$$

if and only if

$$
s \in\left\{z \in \mathbb{C}:\left|z-\left(n-\frac{1}{2}\right)\right|=n-\frac{1}{2}, n \in \mathbb{Z}\right\} .
$$

Figure 5 shows that these circles match the zero trajectories of $f_{ \pm}(s, \alpha)$ very well. 


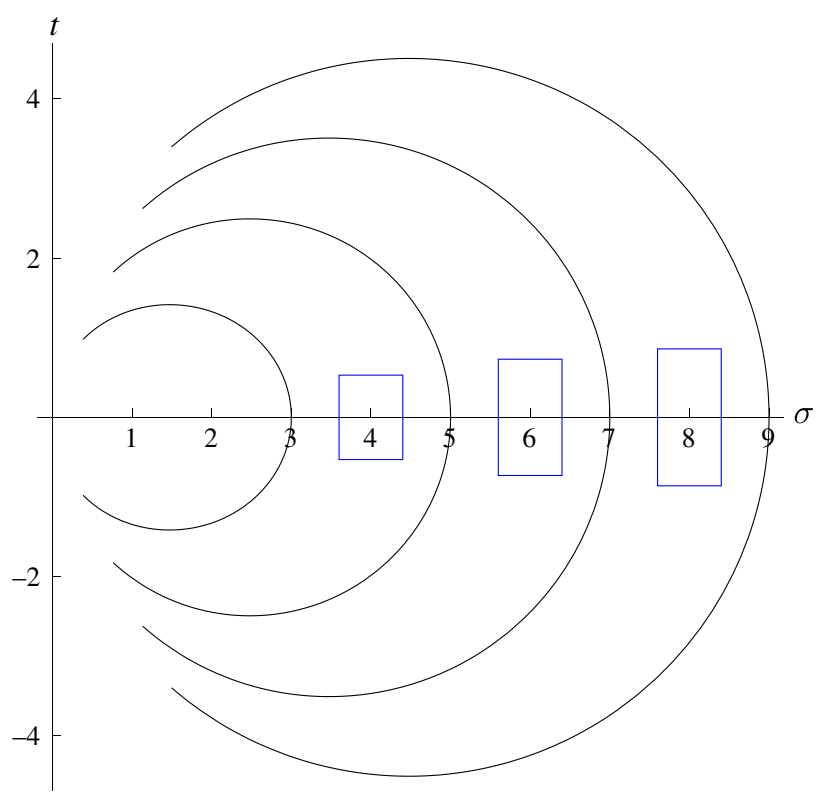

Figure 4 . The blue rectangles are the explicitly calculated zero-free regions for $f_{ \pm}(s, \alpha), \alpha \in(0,1)$, in the $s$-plane. The black curves are the zero trajectories $z(\alpha)$ of $f_{ \pm}(z(\alpha), \alpha)=0$, where $0.001<\alpha<0.999$. The blue boxes are given by the choice $X=0.4<\frac{1}{2}$ and an appropriate $Y$, such that $J(n, X, Y)<1$, see Theorem 5.1. In fact, the triples $(n, X, Y)=(1,0.4,0.53),(2,0.4,0.73),(3,0.4,0.86)$ satisfy the conditions.

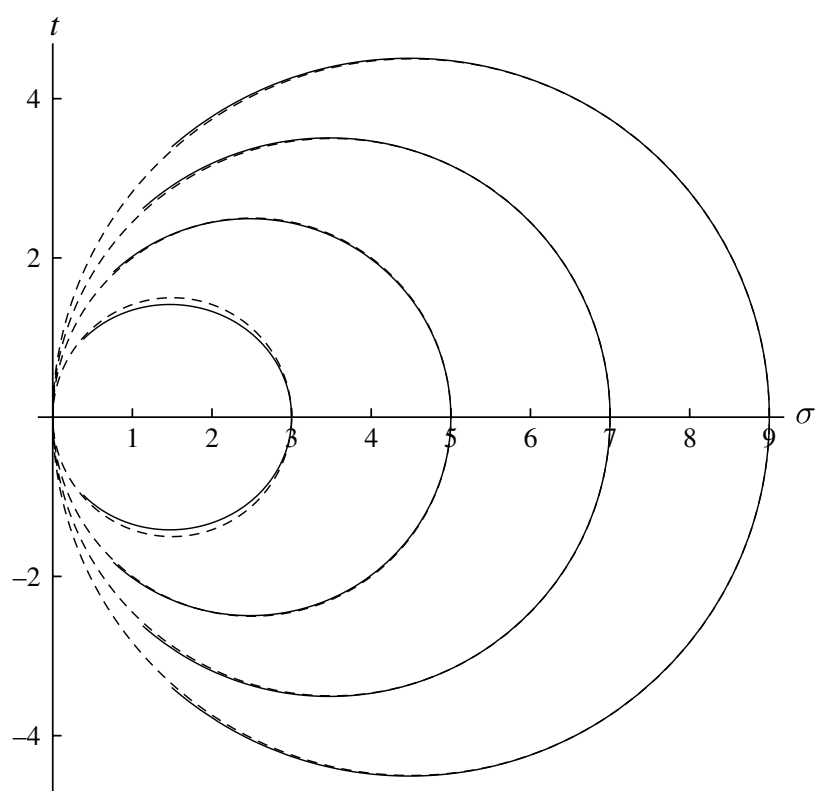

Figure 5. The dashed curves (circles) are the zero trajectories of $1-e^{ \pm i \pi s}(\alpha /(1-\alpha))^{s}$, $0<\alpha \leqslant 1$, in the s-plane. The black curves are the zero trajectories $z(\alpha)$ of $f_{ \pm}(z(\alpha), \alpha)=0$, for $0.001<\alpha<0.999$. 


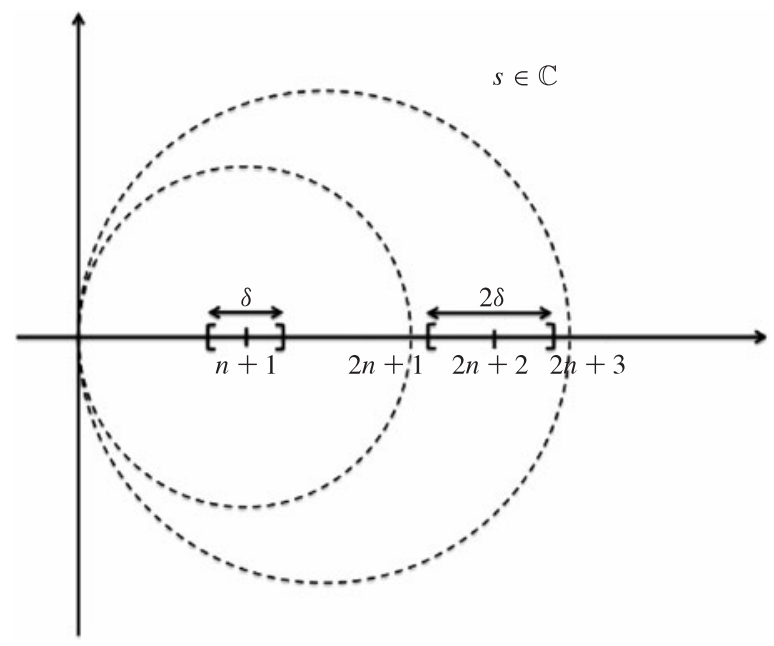

Figure 6. The dashed lines are two adjacent zero trajectories of $f_{ \pm}(s, \alpha)$. The crescent-shaped region can be bounded by circles of radius $n+1 \pm \delta / 2$ with center $n+1 \pm \delta / 2$.

Now let $n \in \mathbb{N}$. In view of Figure 5 , we aim at finding crescent-shaped zero-free regions which lie between two adjacent zero trajectories, see Figure 6. These crescent-shaped regions have the form

$$
Z F\left(n, \sigma_{0}\right):=\left\{s:\left|s-\frac{2 n+2+\delta}{2}\right|=\frac{2 n+2+\delta}{2}, 0<|\delta| \leqslant \delta_{0}, \sigma_{0} \leqslant \sigma \leqslant 2 n+2+\delta_{0}\right\},
$$

where $\delta_{0}<1$ defines the width of the crescent along the real axis and where $2<\sigma_{0}:=\sigma_{0}(n)<$ $2 n+2+\delta_{0}$ is a lower bound for the extent of the crescent. The quantity $\sigma_{0}$ will be specified below.

For $s \in Z F\left(n, \sigma_{0}\right)$, we observe that

$$
\left(\sigma-\frac{2 n+2+\delta}{2}\right)^{2}+t^{2}=\left(\frac{2 n+2+\delta}{2}\right)^{2}
$$

Hence,

$$
t= \pm \sqrt{\sigma(2 n+2+\delta-\sigma)}
$$

which gives

$$
\left|e^{ \pm i \pi s}\left(\frac{\alpha}{1-\alpha}\right)^{s}\right|=\exp \left(\sigma \ln \frac{\alpha}{1-\alpha} \mp \operatorname{sgn}(t) \pi \sqrt{\sigma(2 n+2+\delta-\sigma)}\right)
$$

and

$$
\arg \left(e^{ \pm i \pi s}\left(\frac{\alpha}{1-\alpha}\right)^{s}\right)= \pm \pi \sigma+\operatorname{sgn}(t) \sqrt{\sigma(2 n+2+\delta-\sigma)} \ln \frac{\alpha}{1-\alpha} .
$$

Here, we defined $\operatorname{sgn}(t):= \pm 1$ according to whether $t>0$ or $t<0$, and $\operatorname{sgn}(0):=0$.

As we did in $\S 5$, we prove that $\alpha^{s} f_{ \pm}(s, \alpha) \neq 0$ by distinguishing two cases: (i) the modulus (23) is not close to one; and (ii) (23) is close to one but the argument (24) is not close to $\pi(\bmod 2 \pi)$.

Assume $s \in Z F\left(n, \sigma_{0}\right)$. In view of (23), we see that

$$
\frac{1}{2} \leqslant\left|e^{ \pm i \pi s}\left(\frac{\alpha}{1-\alpha}\right)^{s}\right| \leqslant \frac{3}{2}
$$




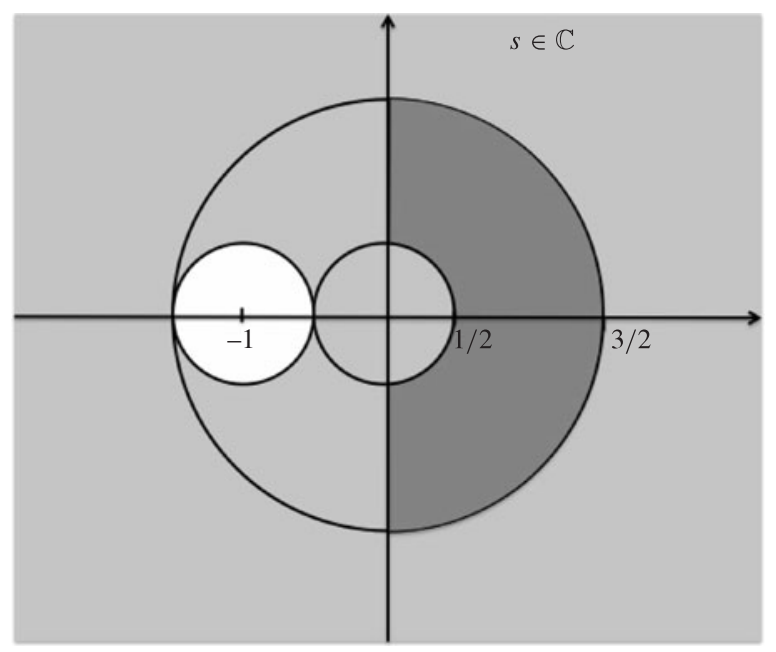

Figure 7. The desired inequality (30) is illustrated by the light grey area. The dark grey area is implied by (25) and (27). It is now obvious that this area has no common points with the white circle $B_{1 / 2}(-1)$, which has to be excluded to satisfy (30).

holds if and only if

$$
\ln \frac{\alpha}{1-\alpha}=\frac{\varepsilon \pm \operatorname{sgn}(t) \pi \sqrt{\sigma(2 n+2+\delta-\sigma)}}{\sigma} \text { for } \ln \frac{1}{2} \leqslant \varepsilon \leqslant \ln \frac{3}{2} .
$$

Equations (25) and (26) imply the following equivalences: the inequalities in (25) and the inequality

$$
\left|\arg \left(e^{ \pm i \pi s}\left(\frac{\alpha}{1-\alpha}\right)^{s}\right)\right| \leqslant \frac{\pi}{2}(\bmod 2 \pi)
$$

are satisfied if and only if (26) and

$$
\left| \pm \pi \pm \pi \delta+\operatorname{sgn}(t) \varepsilon \sqrt{\frac{2 n+2+\delta-\sigma}{\sigma}}\right| \leqslant \frac{\pi}{2} \quad(\bmod 2 \pi)
$$

are valid. Recalling the ranges for $\delta$ and $\varepsilon,(28)$ is true if, for instance,

$$
\sigma \geqslant \frac{2 n+2+\delta_{0}}{\mu}=: \sigma_{1}
$$

where

$$
\mu_{0}:=1+\left(\frac{\ln 2}{\pi}\right)^{2}<\mu<1+\left[\frac{\pi}{\ln 3 / 2}\left(\frac{3}{2}-\delta_{0}\right)\right]^{2}=: \mu_{1} .
$$

The value for the lower bound $\mu_{0}$ for $\mu$ will become clear below.

We claim that for $s \in Z F\left(n, \sigma_{1}\right)$ the inequality

$$
\left|1+e^{ \pm i \pi s}\left(\frac{\alpha}{1-\alpha}\right)^{s}\right| \geqslant \frac{1}{2}
$$

is true for all $0<\alpha<1$. Indeed, if for a given $s \in Z F\left(n, \sigma_{1}\right)$ there is no $\alpha$ for which (26) is satisfied, then (30) is automatically true. On the other hand, if there exists an $\alpha$ so that (26) (or equivalently (25)) holds, then it follows from the definition of $\sigma_{1}$ that (27) is satisfied and therefore (30) is true. For an illustration of the considered sets, see Figure 7. 


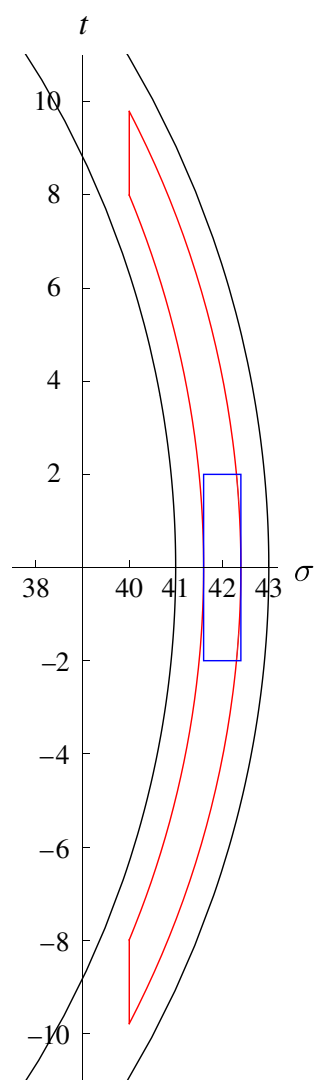

FiguRE 8. Crescent-shaped (red) and rectangular (blue) zero-free regions near $s=42$ of $f_{ \pm}(z(\alpha), \alpha)=0$ with $0<\alpha<1$. The calculations are based on the values $J(20,0.4,2)<1$ for the blue rectangle (cf. Theorem 5.1) and on $\operatorname{Er}(20,40.02)<\frac{1}{2}$ for the crescents. The black curves are the zero trajectories $z(\alpha), f_{ \pm}(z(\alpha), \alpha)=0$.

Next, we consider the error term (20) in the approximation of $\alpha^{s} f_{ \pm}(s, \alpha)$ by $\left(1+e^{ \pm i \pi s}\right)$ $(\alpha / 1-\alpha)^{s}$. In view of $(22)$, we observe that for $s \in Z F\left(n, \sigma_{0}\right)$ the error term

$$
\left(1+e^{\mp \pi t}\right)\left(3^{-\sigma}+\frac{2^{-\sigma}}{\sigma-1}\right)
$$

is less than $1 / 2$ provided we can find a $\sigma_{0} \geqslant \sigma_{1}$ so that

$$
\operatorname{Er}\left(n, \sigma_{0}\right):=\left(1+e^{\pi \sqrt{\sigma_{0}\left(2 n+2+\delta_{0}-\sigma_{0}\right)}}\right)\left(3^{-\sigma_{0}}+\frac{2^{-\sigma_{0}}}{\sigma_{0}-1}\right)<\frac{1}{2} .
$$

The above inequality together with (30) imply then that $f_{ \pm}(s, \alpha) \neq 0$, for all $s \in Z F\left(n, \sigma_{0}\right)$ and all $0<\alpha<1$. To establish the existence of such a $\sigma_{0}$, we use the fact that $\sigma_{0}>2$ and the estimate

$$
3^{-\sigma_{0}}+\frac{2^{-\sigma_{0}}}{\sigma_{0}-1} \leqslant 2 \cdot 2^{-\sigma_{0}}
$$

to show that

$$
\operatorname{Er}\left(n, \sigma_{0}\right) \leqslant\left(1+e^{\pi \sqrt{\sigma_{0}\left(2 n+2+\delta_{0}-\sigma_{0}\right)}}\right) \cdot 2 \cdot 2^{-\sigma_{0}}<\frac{1}{2} .
$$



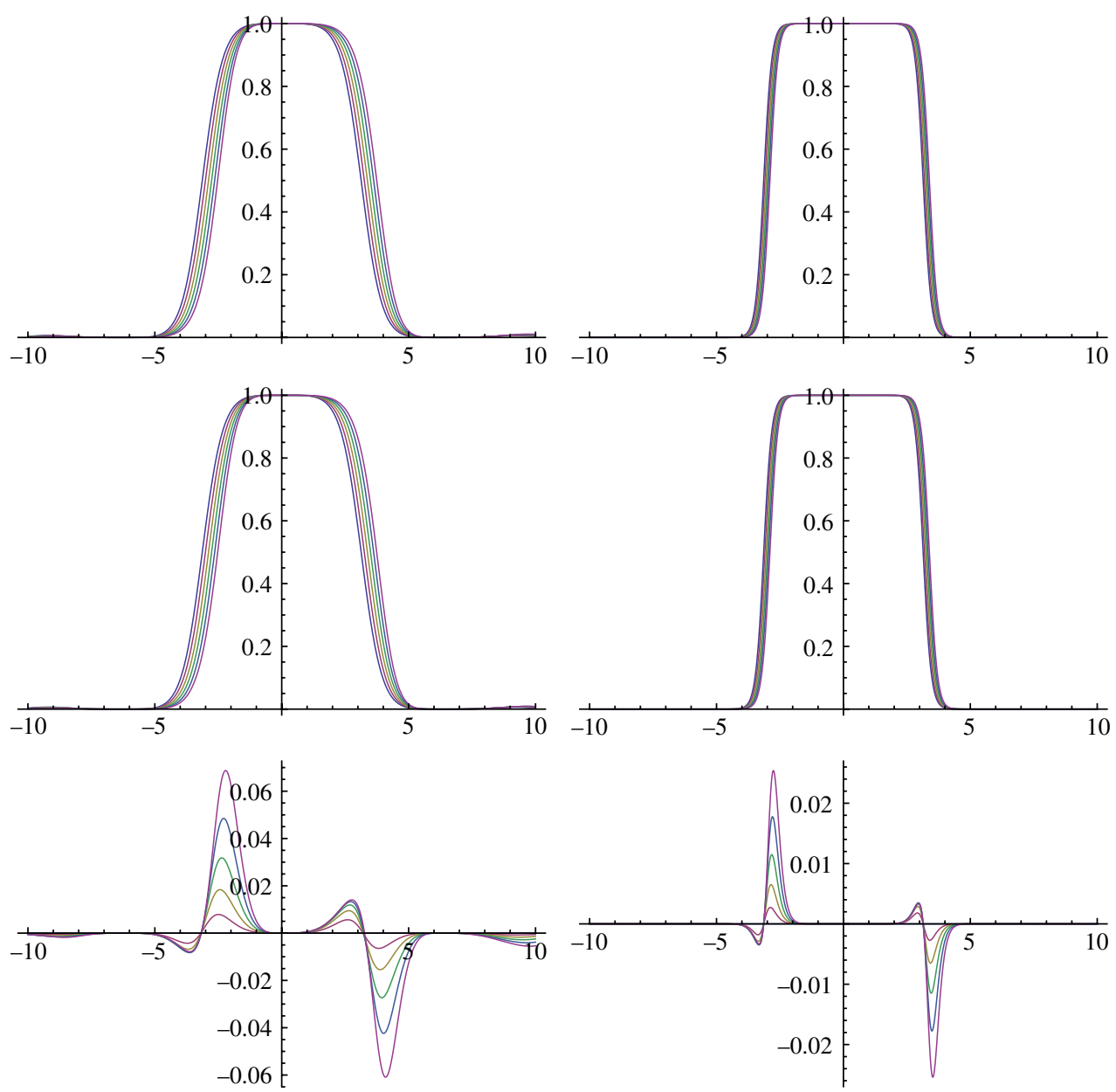

FIGURE 9. Fourier representation of the interpolating splines of complex order for $s=4+k 0.1 i$ (left column) and $s=10+k 0.1 i$ (right column), $k=0,1, \ldots, 5$. Upper row: absolute values, center: real parts and lower row: imaginary parts.

For this purpose, we set $\sigma_{0}:=r \sigma_{1}$, where $1 \leqslant r \leqslant \mu$, use $(29)$, and define a continuous function $\Delta:[1, \mu] \rightarrow \mathbb{R}$ by

$$
\Delta(r):=1+e^{\pi \sigma_{1} \sqrt{r(\mu-r)}}-2^{\sigma_{1} r-2} .
$$

Now, $\Delta(\mu)=2-2^{\sigma_{1} \mu-2}=2-2^{2 n+\delta_{0}}<0$. On the other hand,

$$
\begin{aligned}
\Delta(1) & =1+e^{\pi \sigma_{1} \sqrt{\mu-1}}-2^{\sigma_{1}-2}=1+e^{\pi \sigma_{1} \sqrt{\mu-1}}\left(1-e^{\left(\sigma_{1}-2 / \ln 2-\pi \sigma_{1} \sqrt{\mu-1}\right.}\right) \\
& =1+e^{\pi \sigma_{1} \sqrt{\mu-1}}\left(1-e^{-\left(\sigma_{1}-1\right)(\pi \sqrt{\mu-1}-\ln 2)-(\pi \sqrt{\mu-1}+\ln 2)}\right)>0,
\end{aligned}
$$

since $\mu>1+((\ln 2) / \pi)^{2}$. Hence, there exists a $r_{0} \in(1, \mu)$ so that $\Delta\left(r_{0}\right)=0$. Now choose $\mu \in\left(\mu_{0}, \mu_{1}\right)$ so that $\sigma_{0}:=\sigma_{1} r_{0}>2$. Then $\operatorname{Er}(n, \sigma)<1 / 2$, for all $\sigma \geqslant \sigma_{0}$ and $n \in \mathbb{N}$.

Thus we have proved the following result.

Theorem 6.1. Let $n \in \mathbb{N}$ and let $0<\delta_{0}<1$. Then there exists a $\sigma_{0} \in\left(2,2 n+2+\delta_{0}\right)$ so that for all $s \in Z F\left(n, \sigma_{0}\right)$ and all $0<\alpha<1, f_{ \pm}(s, \alpha) \neq 0$. 

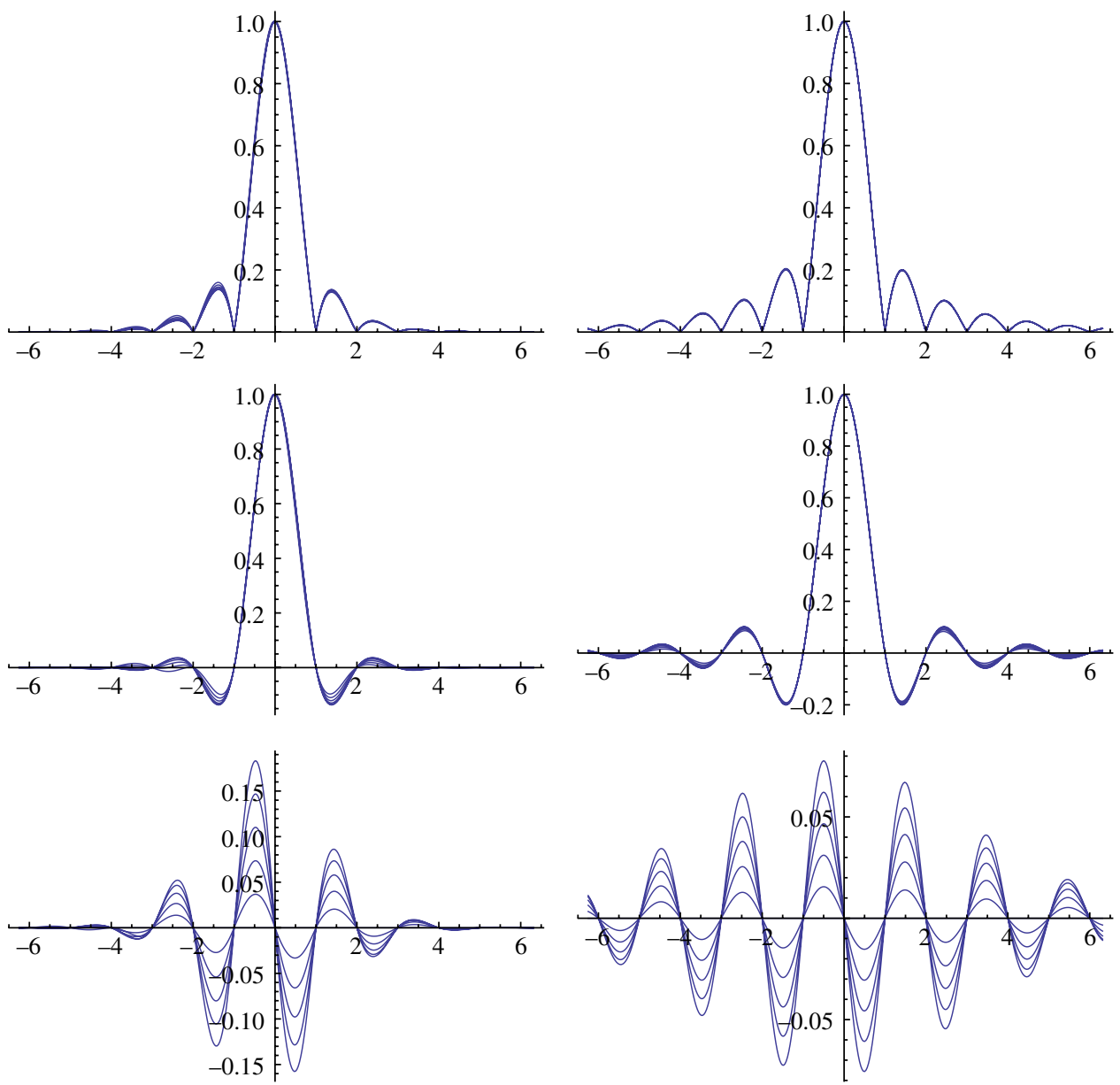

FiguRE 10. Time domain representation of the interpolating splines of complex order for $s=4+k 0.1 i$ (left column) and $s=10+k 0.1 i$ (right column), $k=0,1, \ldots, 5$. Upper row: absolute values, center: real parts and lower row: imaginary parts.

\section{Fundamental cardinal splines of complex order}

The previous sections now provide us with a means to construct a fundamental cardinal spline of complex order

$$
L_{s}:=\sum_{k \in \mathbb{Z}} c_{k}^{(s)} B_{s}(\bullet-k)
$$

solving the interpolation problem (5) for specific values of $s \in \mathbb{C}$.

Theorem 7.1. Suppose that $B_{s}$ is a B-spline of complex order $s$ with $\operatorname{Re} s>2$. Assume that $s$ lies in one of the following regions:

(i) $s \in S(n, X, Y)($ see (17)); or

(ii) $s \in Z F\left(n, \sigma_{0}\right)($ see $(21))$.

Then

$$
L_{s}(x):=\frac{1}{2 \pi} \int_{\mathbb{R}} \frac{\omega^{-s} e^{i \omega x} d \omega}{\zeta(s, \omega / 2 \pi)+e^{-i \pi s} \zeta(s, 1-\omega / 2 \pi)}
$$


is a fundamental interpolating spline of complex order $s$ in the sense that

$$
L_{s}(m)=\delta_{m, 0} \quad \text { for all } m \in \mathbb{Z} .
$$

The Fourier inverse in (31) holds in the $L^{1}$ and $L^{2}$ sense.

For an illustrative example of a fundamental interpolating spline of complex order $s$, see Figures 9 and 10.

\section{Concluding remarks}

If we specify values for $s$ or $\alpha$ then more can be said about zero-free regions. For instance, if $\alpha$ is sufficiently far from zero and one, then one can prove the non-vanishing of $f_{ \pm}(s, \alpha)$ for all sufficiently large $|t|$. This can be proved by using estimates like (11). Moreover, for $\alpha=\frac{1}{2}$ there are no further zeros other than $s \in 2 \mathbb{N}+1$, a fact which follows immediately from the factorization

$$
f_{ \pm}\left(s, \frac{1}{2}\right)=\zeta\left(s, \frac{1}{2}\right)(1+\exp ( \pm i \pi s))=0 .
$$

And, as we have seen in the proof of Theorem 4.1, there are no further real zeros. It seems that many techniques from the theory of zeta functions can be applied to deduce information on the distribution of zeros of $f_{ \pm}$. (See [2].)

Acknowledgement. The second author is partially supported by grant No. MIP-066/2012 from the Research Council of Lithuania.

\section{References}

1. C. K. Chui, An Introduction to Wavelets (Academic Press, San Diego, 1992).

2. A. Laurinčikas and R. Garunkštis, The Lerch Zeta-Function (Kluwer, 2002).

3. B. Forster, T. Blu and M. Unser, 'Complex B-splines', Appl. Comput. Harmon. Anal. 20 (2006) 281-282.

4. B. Forster and P. Massopust, 'Statistical encounters with complex B-Splines', Constr. Approx. 29 (2009) no. 3, 325-344.

5. P. Massopust and B. Forster, 'Multivariate complex B-splines and Dirichlet averages', J. Approx. Theory 162 (2010) 252-269.

6. I. J. Schoenberg, Cardinal spline interpolation, CBMS-NSF Regional Conference Series in Applied Mathematics 12 (SIAM, Philadelphia, 1973)

7. R. SpIRA, 'Zeros of Hurwitz zeta functions', Math. Comput. 30 (1976) no. 136, 863-866.

B. Forster

Fakultät für Informatik und Mathematik

Universität Passau

Innstr. 33, 94032 Passau

Germany

and

Institut für Biomathematik und Biometrie

Helmholtz Zentrum München

Ingolstädter Landstraße 1

85764 Neuherberg

Germany

brigitte.forster@uni-passau.de

\section{R. Garunkštis}

Faculty of Mathematics and Informatics

Vilnius University

Naugarduko 24, 03225 Vilnius

Lithuania

ramunas.garunkstis@mif.vu.lt 
P. Massopust

Institut für Biomathematik und Biometrie Helmholtz Zentrum München

Ingolstädter Landstraße 1

85764 Neuherberg

Germany

and

Zentrum Mathematik, M6

Technische Universität München

Boltzmannstr. 3, 85747 Garching

Germany

massopust@ma.tum.de
J. Steuding

Department of Mathematics

Würzburg University

Am Hubland, 97074 Würzburg

Germany

steuding@mathematik.uni-wuerzburg.de 